PRAMANA

- journal of

(C) Indian Academy of Sciences

Vol. 58, No. 2

physics

February 2002

pp. $149-153$

\title{
Some open problems in the physics of disordered systems
}

\author{
T V RAMAKRISHNAN \\ Department of Physics, Indian Institute of Science, Bangalore 560 012, India
}

\begin{abstract}
Some problems in the physics of disordered systems are pointed out; most of these arise from experiments.
\end{abstract}

Keywords. Disordered systems; electron localization; metal insulator transitions.

PACS Nos 71.55.Jv; 71.23.An; 71.30.+h

I would like to outline here a few problems where disorder is involved, since this is one of the many areas to which N Kumar has contributed greatly.

The first problem, considered one of the great unsolved problems in condensed matter physics, is the formation of a glassy state either without quenched disorder (e.g. window glass) or with it (e.g. spin glass). Is localization of some kind the primary cause? Interestingly, early ideas on spin glasses had a stream [1] which explored the connection between the disorder in the exchange coupling $J_{i j}$, the energy regions in the density of states for such a $J_{i j}$ distribution for which the eigenvalues are localized or extended, and the spin glass transition. This was just before the great progress in both the theory of spin glasses and of localization, which has taken place along nonintersecting paths. In spite of insightful phenomenology for glasses, e.g. the idea of 'fragility' [2] the large amount of numerical work supporting the idea of a phase transition in spin glasses [3] as well as on the nature of local energy minima and energy landscapes in glasses [4] and the development of replica symmetry breaking theories for both spin glasses [5] and glasses [6] it is generally believed that we do not have a comprehensive, deep understanding of glassy systems.

I raise here again the possibility that localization effects are the key. In a system with a fixed total energy (microcanonical ensemble), there are many configurations with total energy lying between say, $E$ and $E+\mathrm{d} E$. The local energy density $\varepsilon(\vec{r})$ fluctuates from site to site, but integrates over the system volume to within $E$ and $E+\mathrm{d} E$. The notion of temperature derives from the Boltzmann approach of counting the total number of such configurations and equating its logarithmic derivative with respect to energy with $\left(1 / k_{\mathrm{B}} T\right)$. The idea of thermal equilibrium originates from the assumption that all these configurations can be reached, one from another, in a 'short' time. Now if the fluctuations in $\varepsilon(\vec{r})$ are very large either because of quenched disorder, or because of the effect annealed disorder has over a period of time, the mixing of configurations, namely a kind of diffusion of local energy fluctuations with total energy conserved, will take place only over very long times. For times less than this, one does not have the idea of temperature. The question is whether this kind of dynamic 'absence of diffusion' approach to configurational localization can 
give us a deeper insight into the spin glass transition and the glassy state. More generally one would like to know whether such an approach, if developed addresses the question of the Gibbsian distribution and the idea of temperature.

I now mention a few disorder/localization related questions that arise from experiments. Some of these are well-known, others are not. Perhaps the best known is the observation of Kravchenko and others [7] that there is a metallic phase in two dimensions, and that there is a continuous quantum phase transition to the insulating phase as the areal electron density is decreased. Though there is a large body of experimental results [8] supporting this idea, namely that in high mobility $\left(\mu \tilde{>} 10^{4} \mathrm{~cm}^{2} / \mathrm{s}\right)$ inversion layer electronic systems there is a metal to insulator transition at $r_{s} \tilde{>} 20$ with a diverging correlation length at $T=$ 0 . There are experimental [9] and theoretical [10] arguments that this transition is an artefact, and that at the lowest temperatures, one always tends to an insulating state as is expected for a noninteracting collection of electrons in a random potential [11]. In this picture the metallic behaviour is attributed to a decrease in electron scattering from traps as these ionized impurities bind electrons with decreasing temperature [10]. Clearly the most immediate necessity is to factor out these effects, and to devise experiments where they do not mask the transition if it exists which is being done. My impression is that there is a residual metal insulator transition. This possiblity has inspired a number of theoretical explanations [12]. Some of these recognize the fact that the transition takes place for $r_{s} \gg 1$, so that typically the Coulomb interaction energy is an order of magnitude larger than the kinetic energy. The conventional many-body, Fermi liquid approach which treats the former qualitatively as a perturbation is not appropriate. However, we do not know how to describe a quantum electron fluid when the Coulomb interaction energy is the larger term. The question is whether such an approach, e.g. a quantum $(T=0)$ Ginzburg-Landau functional for strong interaction and with disorder, can be intuited or derived, and used for looking into the nature of the Wigner crystal/glass to electron fluid transition at $T=0$. Can this functional include 'weak' localization and diffusion effects? Can it also account for the breakdown of screening?

Another (longstanding) major experimental problem is the transition of a superconductor to an insulator, e.g. in two dimensions, as disorder increases [13]. Following the work of Fisher et al [14] it is conventional to think of this as a problem in boson localization, the effective charged bosons being Cooper pairs which form a superfluid. The approach to the transition is based on the idea that vortices (topological defects in the pair field) which are pinned for small disorder, proliferate and form a vortex superfluid for large disorder, and that the latter phase is insulating. The description of the $T=0$ superfluid insulator transition is in the image of a classical clean system phase transition with disorder or interaction replacing the temperature [14]. In this picture, electronic degrees of freedom, effects of disorder such as Anderson localization, electron diffusion (diffusons), and dissipation are not believed to be essential. However, many recent experiments [15] suggest that the scaling predicted by the boson localization theory breaks down at low temperatures, implying that dissipation effects are important. Further, (magneto) transport [16] and tunnelling [17] measurements suggest that low (zero) energy electronic excitations are present as well as important. Thus there seems to be a need to bring back electrons and disorder in this problem.

A third well-known subfield of more recent origin is related to the suggestion, based on transport measurements in disordered (mesoscopic) conductors that the decoherence rate due to inelastic collisions does not vanish as $T \rightarrow 0$ [18]. This is an area of great potential 
significance, since it affects the notion of a single nondegenerate quantum many-electron ground state for metals, and the nature of elementary excitations therefrom. This is a field of considerable controversy; while most experimental results suggest that indeed the inelastic decoherence rate does not go to zero as $T \rightarrow 0$, [18] others are not in agreement. A theoretical argument for such a decoherence [19] is most likely invalid [20]. A large area rich in novel phenomena, where the role of disorder (intrinsic?) disorder is not clear, is the physics of manganites [21] which show colossal magnetoresistance. Over a wide doping range, the compounds $R e_{1-x} A_{x} \mathrm{MnO}_{3}$, where $R e$ and $A$ are rare earth and alkaline earth ions respectively, show a paramagnetic insulator to ferromagnetic metal transition on lowering the temperature. It was suggested [22] that the $e_{g}$ electrons in the background of disordered $t_{2 g}$ spins and consequent random hopping (due to double exchange) are Anderson localized, and that this is the origin of the paramagnetic phase. Subsequent detailed calculations [23] do not bear this out in that the associated disorder is too small, so that the paramagnetic phase must have a different origin as also the insulator-metal transition. However, there is another ubiquitous phenomenon in these systems, namely the coexistence of localized (insulating, Jahn Teller distorted? orbitally or charge ordered?) and metallic regions ('phases') over a broad range of temperature $T$ and concentration $x$ [24]. The origin of this is unclear. Since these regions are often large, of micron size, the idea that one has conventional phase separation, with regions of different ion/electron concentration $x_{\mathrm{m}}$ (metallic), and $x_{\mathrm{co}}$ (charge ordered) coexisting seems untenable, there being experimental evidence that the $A$ ion concentration is uniform. In that case, there would be large destabilizing Coulomb repulsion in each region if $x_{\mathrm{m}}$ and $x_{\mathrm{co}}$ have different electron density. So the natural conclusion is that while $x$ is constant as is the $e_{g}$ electron concentration, some other degree of freedom must have variations on a length scale which can be up to a few microns. What is this degree of freedom? What is the effect on $e_{g}$ electrons of disorder in this coordinate? How can this disorder have such large length scales?

Finally, I mention a problem where disorder seems to play a 'delocalizing' role. This is in stable quasicrystals, e.g. $\mathrm{Al}_{5 / 8} \mathrm{Cu}_{1 / 4} \mathrm{Fe}_{1 / 8}$. In these systems the electrical conductivity decreases as disorder decreases [25]. The cleanest quasicrystals have a conductivity much smaller (smaller by a factor of ten to hundred) than the Mott minimum metallic conductivity of a metal with the conduction electron density appropriate to these systems. Secondly, as temperature increases, the electrical conductivity increases [26] and in the same way in many systems, e.g. $\Delta \sigma(T)=\sigma(T)-\sigma(0)$ is the same for different compositions around $\mathrm{Al}_{5 / 8} \mathrm{Cu}_{1 / 4} \mathrm{Fe}_{1 / 8}$, and for $\mathrm{Al}_{70.5} \mathrm{Pd}_{22} \mathrm{Mn}_{7.5}$. The Hall coefficient is very large (as if the carrier density were low) and strongly temperature dependent, changing sign with it [27]. There are anomalies in optical conductivity as well [28]. These unusual electronic properties are not well understood; the naive explanation [29] based on a dip or pseudogap in the density of states is inadequate since the dip (experimentally) is by a factor of three or so, whereas the anomalies are qualitatively different (from what is expected with a dip) and quantitatively enormous. One qualitative explanation proposed is of nonclassical electron diffusion in a self-similar structure [30]; this does not come to grips with the facts. Another possibility [31] is that because the Brillouin (Jones) zone has many faces very closely overlapping the free electron Fermi sphere, the Fermi surface is cut up into a large number of small electron and hole surfaces and one has a semi-metal. Disorder, via scattering, connects these pieces with one another, leading to a metal with a large Fermi surface, and lower resistivity. Temperature plays a similar role.

Pramana - J. Phys., Vol. 58, No. 2, February 2002 


\section{TV Ramakrishnan}

To conclude, I have tried to indicate using some real life examples the variety of ways in which disorder crucially affects physical properties of condensed matter systems. Hopefully, N Kumar, who has thought about many of these questions, will find them interesting.

\section{References}

[1] P W Anderson, in Ill-Condensed Matter edited by R Balian, R Maynard and G Toulouse (Northe Holland, Amsterdam, 1979)

[2] For example, see C A Angell, Science 267, 1924 (1995)

[3] See for example, A P Young (ed.) Spin Glasses and Random Fields (World Scientific, Singapore, 1998)

[4] S Sastry, P G Debenedetti and F H Stillinger, Nature 393, 554 (1998)

[5] For a review, see M Mezard, G Parisi and M A Virasaro, Spin Glass Theory and Beyond (World Scientific, Singapore, 1987)

[6] See for example, M Mezard, in More is Different edited by N Phuan Ong and Ravin N Bhatt (Princeton U Press, Princeton, 2001) p. 237

[7] S V Kravchenko, G V Kravchenko, J E Furneaux, V M Pudalov and M D'Iorio, Phys. Rev. B50, 8039 (1994)

[8] E Abrahams, S V Kravchenko and M P Sarachik, briefly review the field in Rev. Mod. Phys. 73, $251(2001)$

[9] M Y Simmons, A R Hamilton, M Pepper, E H Linfield, P D Rose and D A Ritchie, Phys. Rev. Lett. 84, 2489 (2000)

[10] B L Altshuler and D L Maslov, Phys. Rev. Lett. 82, 145 (1999)

[11] E Abrahams, P W Anderson, D C Licciardello and T V Ramakrishnan, Phys. Rev. Lett. 42, 673 (1979)

[12] S Chakravarty, S Kivelson, C Nayak, K Volker - cond-mat 9805383, Qimiao Si and C M Varma, Phys. Rev. Lett. 81, 4951 (1998)

[13] N Markovic et al, cond-mat 9904168

[14] M P A Fisher, P B Weichmann, G Grinstein and D S Fisher, Phys. Rev. B40, 546 (1989)

[15] N Mason and A Kapitulnik, Phys. Rev. Lett. 82, 5341 (1999)

[16] S Y Hsu, J A Chervenak and J M Valles Jr. Phys. Rev. Lett. 75, 132 (1995)

[17] J M Valles, R C Dynes and J P Garno Phys. Rev. Lett. 69, 3567 (1992)

[18] P Mohanty, EMQ Jariwala and RA Webb, Phys. Rev. Lett. 78, 3366 (1997) P Mohanty, Ann. Phys. (Leipzing) 8, 549 (1999)

[19] D S Golubev, A D Zakin and G Schon, cond-mat 0110495

[20] L L Aleiner, B L Altshuler and M G Vavilov, cond-mat 0110545

[21] See e.g. Colossal Magnetoresistence Oxides edited by Y Tokura, (Gordon and Breach, London, 1999)

[22] C M Varma, Phys. Rev. B54, 7328 (1996)

[23] Q Li, J Zang, A R Bishop and C M Soukoulis, Phys. Rev. B56, 15090 (1997)

[24] M Uehara, S Mori, C H Chen and S W Cheong, Nature 399, 560 (1999) See also E Dagotto, T Hotta nad A Moreo, Phys. Rep. 344, 1 (2001)

[25] See C Janot, Quasicrystals: A Primer, 2nd ed. (Oxford University Press, New York, 1994)

[26] D Mayou, C Berger, F Cyrot-Lackmann, T Klein and D Lanco, Phys. Rev. Lett. 70, 3915 (1993)

[27] F S Pierce, P A Bancel, B D Biggs, Q Guo and S J Poon, Phys. Rev. B47, 5670 (1993)

[28] X Wu, C C Homes, S E Burkov, T Tinusk, F S Pierce, S J Poon, S L Cooper and M A Karlow, J. Phys. Condens. Matter 5, 5975 (1993) 


\section{Physics of disordered systems}

[29] P Bancel and P A Heiney, Phys. Rev. B33, 7917 (1986)

T Fujiwara and T Yokokawa, Phys. Rev. Lett. 66, 333 (1991)

[30] B Passaro, C Sire and V Benza, Phys. Rev. B46, 13757 (1992)

[31] T V Ramakrishnan (to be published) 